\title{
Magnetic relaxation in metallic films: Single and multilayer structures
}

\author{
B. Heinrich, ${ }^{\text {a) }}$ R. Urban, and G. Woltersdorf \\ Simon Fraser University, 8888 University Drive, Burnaby, British Columbia V5A 1S6, Canada
}

The intrinsic magnetic relaxations in metallic films will be discussed. It will be shown that the intrinsic damping mechanism in metals is caused by incoherent scattering of itinerant electron-hole pair excitations by phonons and magnons. Berger [L. Berger, Phys. Rev. B 54, 9353 (1996)] showed that the interaction between spin waves and itinerant electrons in multilayers can lead to interface Gilbert damping. Ferromagnetic resonance (FMR) studies were carried out using magnetic single and double layer films. The FMR linewidth of the Fe films in the double layer structures was found to always be larger than the FMR linewidth measured for the single Fe films having the same thickness. The increase in the FMR linewidth scaled inversely with the film thickness, and was found to be linearly dependent on the microwave frequency. These results are in agreement with Berger's predictions. (C) 2002 American Institute of Physics. [DOI: 10.1063/1.1447215]

\section{INTRODUCTION}

It is currently of considerable interest to acquire a thorough understanding of the spin dynamics and magnetic relaxation processes in the nanosecond time regime.

The spin dynamics in the classical limit is given by the Gilbert equation of motion where the relaxation term is expressed by the well known Gilbert damping torque $\left(G / \gamma^{2} M_{s}^{2} \mathbf{M} \times(\partial \mathbf{M} / \partial t) .{ }^{1} M_{s}\right.$ is the saturation magnetization and $\gamma$ is the gyromagnetic ratio.

\section{MAGNETIC RELAXATION PROCESSES IN SINGLE LAYERS}

In metallic films the magnetic damping can be affected by eddy currents. The role of eddy currents in thin films can be estimated by evaluating the effective Gilbert damping that accompanies precession of the magnetic moment around its internal magnetic field. The contribution of eddy currents in this case can be calculated by integrating Maxwell's equations across the film thickness $d$. This approach is justified when the rate of magnetization precession allows full penetration of the associated rf magnetic field. In this case the effective eddy current Gilbert damping $G_{\text {eddy }}$ (in Gaussian units)

$$
\frac{G_{\text {eddy }}}{\left(M_{s} \gamma\right)^{2}}=\frac{1}{6}\left(\frac{4 \pi}{c}\right)^{2} \sigma d^{2},
$$

where $\sigma$ is the electrical conductivity. For $\mathrm{Fe}, G=0.8$ $\times 10^{8} \mathrm{~s}^{-1}$. For a film thickness of $50 \mathrm{~nm} G_{\text {eddy }}=G$.

Suhl presented calculations for the magnon-phonon Gilbert damping ${ }^{2} G_{\mathrm{ph}}$,

$$
\frac{G_{\mathrm{ph}}}{\gamma}=2 \frac{(1+\nu)^{2} B_{2}^{2}}{E^{2}} \eta \gamma,
$$

\footnotetext{
a)Electronic mail: bheinric@sfu.ca
}

where $\eta$ is the phonon viscosity, $B_{2}$ is the magnetoelastic shear constant, $E$ is the Young's modulus, and $\nu$ is the Poisson ratio. All parameters can be readily obtained except the parameter for the phonon viscosity $\eta$. In the measurements of phonon assisted microwave transmission ${ }^{3}$ on $\mathrm{X}$-tal $\mathrm{Ni}$ slabs, $22 \mu \mathrm{m}$ in thickness, we were able to determine the intrinsic lifetime, $\tau_{\mathrm{ph}}=6.6 \times 10^{-10} \mathrm{~s}$, of phonons which correspond to shear waves at a angular frequency of $\omega=2 \pi$ $\times 10 \mathrm{GHz}$. The phonon viscosity is given by $\eta$ $=c_{44} / \tau_{\mathrm{ph}} \omega^{2}$, where $c_{44}$ is the elastic modulus. For Ni $\eta$ $=3.4$ (in CGS). Using the elastic and magnetic properties of $\mathrm{Ni}$ results in a phonon Gilbert damping coefficient which is 30 times smaller than the measured intrinsic damping of $\mathrm{Ni}$, $G=2.4 \times 10^{8} \mathrm{~s}^{-1}$. In $\mathrm{Fe}$ the intrinsic damping $G=0.8$ $\times 10^{8} \mathrm{~s}^{-1}$ is smaller and $M_{s}$ is higher compared to in $\mathrm{Ni}$, and consequently the direct magnon-phonon damping in $\mathrm{Fe}$ is only 6 times smaller than the intrinsic damping. Clearly, direct magnon-phonon scattering is unimportant in high quality metallic films. The above estimates were carried out for small precessional angles. It is often said that the large precession angles involved in the magnetization reversal process can significantly enhance direct magnon-phonon relaxation. The results of Leaver and Vojdani ${ }^{4}$ contradict this assumption. In domain wall mobility measurements using crystalline $\mathrm{Ni}$ platelets (200 $\mathrm{nm}$ thick) the motion of domain walls involves a precessional angle of $180^{\circ}$, and yet the domain wall mobility was explained well by the Gilbert damping obtained from ferromagnetic resonance (FMR).

In the 1970's it was shown that the intrinsic magnetic relaxation in metals is different from that in magnetic insulators. The Gilbert damping in metals is caused by incoherent scattering of electron-hole pair excitations by phonons and magnons. The electron-hole excitations involve three particle scattering (see Fig. 1). The electron-hole pair excitations are either accompanied by spin flip or the spin remains unchanged. Spin-flip electron-hole excitations $\left(\sigma=+\frac{1}{2}, \sigma^{\prime}\right.$ 


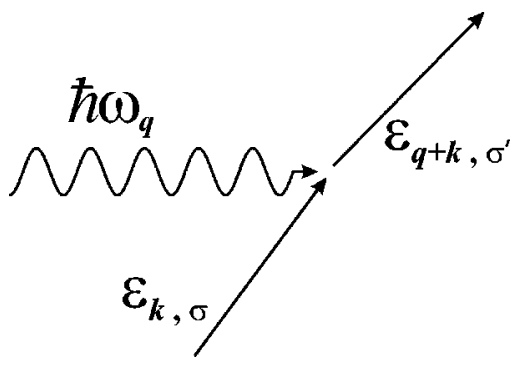

FIG. 1. Spin wave with energy $\hbar \omega_{q}$ collides with an itinerant electron with energy $\varepsilon_{k, \sigma}(\sigma$ represents the spin state), and creates an itinerant electron with momentum $\mathbf{k}+\mathbf{q}$ and spin orientation $\sigma^{\prime}$.

$=-\frac{1}{2}$ ) can be caused by the exchange interaction between magnons and itinerant electrons $\left(s-d\right.$ exchange interaction $\left.{ }^{5}\right)$. The total angular momentum in the $s-d$ exchange interaction is conserved. The electron spin conserving scattering $\left(\sigma=\sigma^{\prime}\right)$ is caused by spin-orbit interaction which leads to a dynamic redistribution of electrons in the electron k-momentum space. ${ }^{6}$ The intrinsic damping in pure metals can be expected to have a strong temperature dependence. The temperature dependence of intrinsic damping can be discussed by inspecting the expression for energy conservation of the scattering shown in Fig. 1. The Gilbert damping can be calculated using Fermi's golden rule, which sums up all available states that satisfy the conservation of energy. The incoherent scattering by phonons can be accounted for by broadening the conservation of energy into a Lorenzian:

$$
\frac{\hbar / \tau_{\text {eff }}}{\left(\hbar \omega_{q}+\varepsilon_{k, \sigma}-\varepsilon_{k+q, \sigma^{\prime}}\right)^{2}+\left(\hbar / \tau_{\text {eff }}\right)^{2}},
$$

where the relaxation rate, $1 / \tau_{\text {eff }}$, describes the incoherent scattering with phonons and magnons. ${ }^{5}$ For the spin-flip electron-hole excitations (subscript ${ }_{\text {eff }}$ becomes subscript ${ }_{\text {flip }}$ ) the difference in electron energy, $\varepsilon_{k, \sigma}-\varepsilon_{k+q, \sigma^{\prime}}$, is given by the exchange interaction energy which is much bigger than $\hbar / \tau_{\text {flip }}$. In this case Eq. (3) is proportional to $\tau_{\text {flip }}^{-1}$. The lifetime of the spin-flip electron-hole pair, $\tau_{\text {flip }}$, is enhanced compared to the orbital relaxation time, $\tau_{\text {orb }}$, that enters the conductivity. The reason is that one needs to invoke the spin-orbit interaction to flip the electron spin by phonons. Elliot $^{7}$ showed that $\tau_{\text {flip }}=\tau_{\text {orb }} / \Delta g^{2}$, where $\Delta g$ is the enhancement of the $g$ factor from spin-orbit interaction. In this case the Gilbert damping is proportional to $\tau_{\text {orb }}^{-1}$ and consequently should be proportional to the sample's resistivity. ${ }^{5}$

For electron spin conserving collisions the change in the electron energy can involve a small wave number $q$ of the FMR magnon and can be neglected compared to $\hbar / \tau_{\text {orb }}$. Equation (3) is proportional to $\tau_{\text {orb }}$. In this case the Gilbert damping is proportional to the sample's conductivity. However, spin-orbit coupling also involves scattering with a large change in the electron energy (scattering from $d$ states into $s$ or $p$ states) and then the corresponding Gilbert damping is again proportional to the resistivity as in the case of the $s-d$ exchange mechanism. ${ }^{8,9}$ In pure metals the Gilbert damping due to spin conserving collisions can be significantly increased at low temperatures by an increase in conductivity $\sigma$. Bhaghat et al. observed in their FMR measure- ments eventual saturation of the Gilbert damping with a decrease in temperature. This result was explained by Korenman and Prange ${ }^{10}$ using Eq. (3). They showed that the Gilbert damping saturates for $\tau_{\text {orb }} \rightarrow \infty$ due to electron surfriding effects. By employing ferromagnetic antiresonance (FMAR) in microwave transmission measurements we were able to avoid electron surfriding. We found that in high purity single crystals of $\mathrm{Ni}$ the terms proportional to the conductivity and resistivity contribute equally to intrinsic damping. ${ }^{11}$

\section{MAGNETIC RELAXATION PROCESSES IN MULTILAYERS}

Magnetic multilayers provide a special case in which the dynamic interaction between the itinerant electrons and the magnetic moments in ultrathin films offers new exciting possibilities. It has been shown that magnetization reversal can be driven by a spin polarized current flowing perpendicular to the magnetic layers. ${ }^{12,13}$ This leads, for sufficiently high current densities, to spontaneous magnetization precession and switching phenomena. Theoretical treatment of the nonlocal $s-d$ exchange interaction in systems consisting of two magnetic layers separated by a nonmagnetic spacer was done by Slonczewski ${ }^{14}$ and by Berger. ${ }^{15}$ Berger in his treatment included magnons in the thinner layer. The thicker magnetic layer was in a static state. He showed that this leads to additional exchange torque which is directed towards the internal field, and represents an additional Gilbert-like relaxation term. The exchange relaxation torque is confined to the vicinity of the nonmagnetic/magnetic layer interface. The resulting relaxation torque in magnetic double layers contributes to an additional FMR linewidth, $\Delta H_{\text {add }}$, and is proportional to

$$
\Delta H_{\text {add }} \sim(\Delta \mu+\hbar \omega),
$$

where $\Delta \mu=\Delta \mu_{\uparrow}-\Delta \mu_{\downarrow}$ is the difference in the spin up and spin down Fermi level shifts, and $\omega$ is the microwave angular frequency. The sign of $\Delta \mu$ depends on the direction of dc current passing perpendicular through the film interface, and can lead to magnetization reversal. The second term in Eq. (4) is always positive and proportional to the microwave frequency. This term does not require a dc current crossing the interfaces. It represents interface Gilbert damping. In ultrathin magnetic films the interface torque is shared by all atomic layers. ${ }^{1}$ It follows that the additional FMR linewidth should have a typical $1 / d$ dependence on the magnetic film thickness $d$.

Berger's predictions shown in Eq. (4) are challenging for experimental studies. Recently we carried out such studies. ${ }^{16}$ The studies were done using crystalline magnetic single $\mathrm{Au} /$ $\mathrm{Fe} / \mathrm{GaAs}(001)$ and double layer $\mathrm{Au} / \mathrm{Fe} / \mathrm{Au} / \mathrm{Fe} / \mathrm{GaAs}(001)$ structures. In the double layer structures the thin Fe layers [8, $11,16,21$, and 31 monolayers (ML) in thickness] from single layer structures were separated from a thick Fe layer of 40 ML thickness by a 40 ML thick Au spacer. The thin Fe layers were deposited directly onto semi-insulating epi-ready $\mathrm{GaAs}(001)$ wafers by means of molecular beam epitaxy (MBE). In-plane FMR measurements at 9.5, 24, and $36 \mathrm{GHz}$ were employed to determine the in-plane fourfold and uniaxial magnetic anisotropies, $K_{1}$ and $K_{u}$, and the effective 


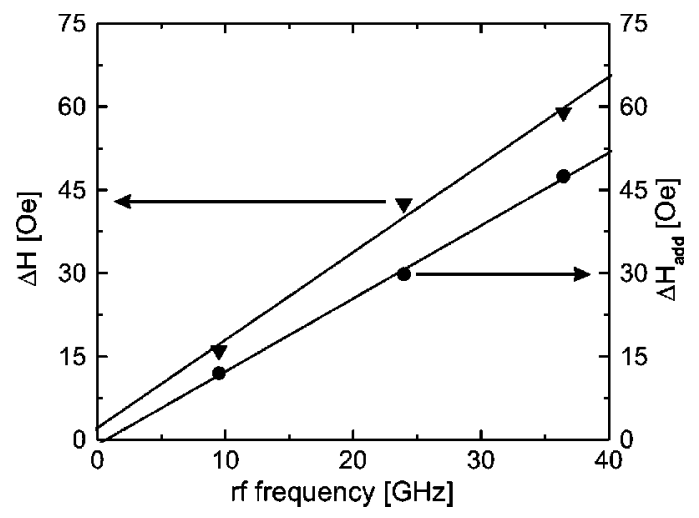

FIG. 2. FMR linewidth, $\Delta H$, as a function of the microwave frequency, $f$, $\Delta H(\omega)=\Delta H(0)+1.16(\omega / \gamma)\left(G / \gamma M_{s}\right)$. ( $\left.\mathbf{\nabla}\right)$ Fe film $16 \mathrm{ML}$ thick in the single layer structure. (๑) Additional contribution, $\Delta H_{\text {add }}$, due to nonlocal damping in the corresponding double layer structure.

demagnetizing field perpendicular to the film's surface, $4 \pi M_{\text {eff }}$, as a function of thickness. The Fe ultrathin films grown on $\operatorname{GaAs}(001)$ have magnetic properties that are nearly equal to those in bulk Fe, modified only by sharply defined interface anisotropies, indicating that the Fe layers are of high crystalline quality with well defined interfaces. The interface magnetic anisotropies allowed us to separate the FMR field of the thin layer from that of the thick layer by a sufficient margin. Hence, the FMR measurements of the Fe thin film in double layers were carried out with the magnetic moment of the thick Fe film far from its FMR, thus possessing a negligible precession angle.

The line shape of the FMR peaks is Lorenzian and the FMR linewidths are small and only weakly dependent on the film thickness. The frequency dependence of the FMR linewidth of the single Fe films was found to be linearly dependent on the microwave angular frequency, $\omega$, with a negligible zero frequency offset $\Delta H(0)$ (see Fig. 2). The Gilbert damping and zero frequency offset were found to be $G$ $=(1.3 \pm 0.1) \times 10^{8} \mathrm{~s}^{-1}$ and $\Delta H(0)=2 \pm 3$ Oe, respectively. The reproducible magnetic anisotropies and small FMR linewidths provided us with an excellent opportunity to investigate nonlocal relaxation processes in multilayer films. ${ }^{16}$

The FMR linewidth in the thin films always increased in the presence of a second thick magnetic layer. The difference between the FMR linewidth in magnetic double and single layers was proportional to the microwave frequency, with no appreciable zero frequency offset $\Delta H(0)$. It means that ultrathin Fe films in double magnetic layers acquire an additional Gilbert damping term. The additional Gilbert damping is a robust effect in $\mathrm{Fe} / \mathrm{Au} / \mathrm{Fe}$ structures. For a $16 \mathrm{ML}$ thick Fe film the strength of the interface Gilbert damping, $G$ $=1.05 \times 10^{8} \mathrm{~s}^{-1}$ is comparable to the Gilbert damping in the single layer structure, $G=1.3 \times 10^{8} \mathrm{~s}^{-1}$, (see Fig. 2). The additional FMR linewidth, $\Delta H_{\text {add }}$, followed an inverse dependence on the thin film thickness $d .{ }^{16}$ This means that the additional contribution to the FMR linewidth can be described by interface Gilbert damping. This is in excellent agreement with Berger's prediction of interface nonlocal Gilbert damping in magnetic multilayers.

Experimental verification of interface Gilbert damping proposed by Berger has important implications for spin dynamics in magnetic multilayers. It offers direct access to experimental studies of relaxation torque caused by the transfer of electron angular momentum between the magnetic layers in the presence of electron-electron exchange interaction. It also has a practical implication; one should be able to test the suitability of magnetic unpatterned multilayers for their use in systems employing mesoscopic magnetization precession and switching processes, e.g., in the spin-wave amplification by stimulated emission of radiation (SWASER) device proposed by Berger. ${ }^{15}$

\section{ACKNOWLEDGMENTS}

The authors thank J. F. Cochran, K. Myrtle, and K. Ajdari for their assistance and for valuable discussions during the course of this work. Financial support from the Natural Sciences and Engineering Research Council of Canada (NSERC) and the Canadian Institute for Advanced Research (CIAR) is gratefully acknowledged.

\footnotetext{
${ }^{1}$ B. Heinrich and J. F. Cochran, Adv. Phys. 42, 523 (1993).

${ }^{2}$ H. Suhl, IEEE Trans. Magn. 34, 1834 (1998).

${ }^{3}$ B. Heinrich, J. F. Cochran, and K. Myrtle, J. Appl. Phys. 53, 2092 (1982).

${ }^{4}$ K. D. Leaver and S. Vojdani, J. Appl. Phys. 3, 729 (1970).

${ }^{5}$ B. Heinrich, D. Fraitová, and V. Kamberský, Phys. Status Solidi 23, 501 (1967).

${ }^{6}$ V. Kamberský, Can. J. Phys. 48, 2906 (1970).

${ }^{7}$ R. J. Elliot, Phys. Rev. 96, 266 (1954).

${ }^{8}$ V. Kambersky, Czech. J. Phys., Sect. B 26, 2906 (1976).

${ }^{9}$ J. F. Cochran and B. Heinrich, IEEE Trans. Magn. MAG-16, 660 (1980).

${ }^{10}$ V. Korenman and R. Prange, Phys. Rev. B 6, 1972 (1972).

${ }^{11}$ B. Heinrich, D. J. Meredith, and J. F. Cochran, J. Appl. Phys. 50, 7726 (1979)

${ }^{12}$ J. A. Katine, F. J. Albert, R. A. Buhrman, E. B. Myers, and D. C. Ralph, Phys. Rev. Lett. 84, 3149 (2000).

${ }^{13}$ M. Tsoi, A. G. M. Jansen, J. Bass, W.-C. Chiang, M. Seck, V. Tsoi, and P. Wyder, Phys. Rev. Lett. 80, 4281 (1998).

${ }^{14}$ J. C. Slonczewski, J. Magn. Magn. Mater. 159, 1 (1996).

${ }^{15}$ L. Berger, Phys. Rev. B 54, 9353 (1996).

${ }^{16}$ R. Urban, G. Woltensdorf, and B. Heinrich, Phys. Rev. Lett. 87, 217204 (2001).
} 